\title{
Correction to: High-Throughput Screening for Insulin Secretion Modulators
}

\author{
Michael A. Kalwat
}

Correction to:

Chapter 9 in: Florence Niedergang, Nicolas Vitale and Stéphane Gasman (eds.), Exocytosis and Endocytosis: Methods and Protocols,

Methods in Molecular Biology, vol. 2233,

https://doi.org/10.1007/978-1-0716-1044-2_9

The chapter was inadvertently published with incorrect email address and a word "Indiana" missed out in the affiliation.

The email address has been updated and the word "Indiana" has been included in the affiliation as Lilly Diabetes Center of Excellence, Indiana Biosciences Research Institute, Indianapolis, IN, USA. 